\title{
Comprehensive analysis of patients with Stargardt macular dystrophy reveals new genotype-phenotype correlations and unexpected diagnostic revisions
}

\author{
Jacques Zaneveld, BS 1,2, Sorath Siddiqui, MD³, Huajin Li, MS ${ }^{4}$, Xia Wang, PhD 1,2, Hui Wang, PhD ${ }^{1,2}$,

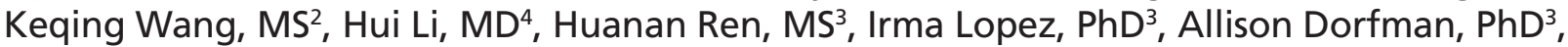 \\ Ayesha Khan, MD³, Feng Wang, BS ${ }^{1,2}$, Jason Salvo, MS ${ }^{1,5}$, Violet Gelowani, BS ${ }^{2}$, Yumei Li, PhD ${ }^{1,2}$, \\ Ruifang Sui, MD, PhD ${ }^{4}$, Robert Koenekoop, MD, $\mathrm{PhD}^{3}$ and Rui Chen, $\mathrm{PhD}^{1,2,5,6}$
}

Purpose: Stargardt macular dystrophy (STGD) results in early central vision loss. We sought to explain the genetic cause of STGD in a cohort of 88 patients from three different cultural backgrounds.

Methods: Next-generation sequencing using a novel capture panel was used to search for disease-causing mutations. Patients with undetermined causes were clinically reexamined and tested for copynumber variations as well as intronic mutations.

Results: We determined the cause of disease in $67 \%$ of our patients. Our analysis identified 35 novel ABCA4 alleles. Eleven patients had mutations in genes not previously reported to cause STGD. Finally, $45 \%$ of our patients with unsolved causes had single deleterious mutations in $A B C A 4$, a recessive disease gene. No likely pathogenic copy-number variations were identified.
Conclusion: This study expands our knowledge of STGD by identifying dozens of novel alleles that cause the disease. The frequency of single mutations in ABCA4 among STGD patients is higher than that among controls, indicating that these mutations contribute to disease. Disease in 11 patients was explained by mutations outside $A B C A 4$, underlining the need to genotype all retinal disease genes to maximize genetic diagnostic rates. Few $A B C A 4$ mutations were observed in our French Canadian patients. This population may contain an unidentified founder mutation. Our results indicate that copy-number variations are unlikely to be a major cause of STGD.

Genet Med advance online publication 4 December 2014

Key Words: clinical genetics; copy-number variations; diagnosis; macular degeneration; vision research

\section{INTRODUCTION}

Stargardt macular dystrophy (STGD) is the most common form of juvenile macular dystrophy, affecting $~ 1$ in 10,000 individuals worldwide. ${ }^{1}$ STGD consists of childhood visual acuity loss, central scotomas on visual field testing, and atrophic maculopathy. The subretinal buildup of oily droplets called lipofuscin in the retinal pigment epithelium cell layer disrupts photoreceptors, leading to photoreceptor death and retinal degeneration. ${ }^{2}$

This disease is particularly devastating because affected individuals lose central vision, which is necessary for common tasks including reading, schooling, driving, and recognizing faces. Most cases of STGD are caused by recessive ABCA4 mutations. "STGD-like" autosomal dominant diseases can be caused by mutations in PROM1 ${ }^{4}$ and ELOVL4 $4^{5}$. Of these three genes, $A B C A 4$ is by far the most frequent cause of disease and the only known cause of recessive STGD. Mutations in ABCA4 also cause the related disorders of cone-rod dystrophy and retinitis pigmentosa $(\mathrm{RP}){ }^{6}$

Ongoing gene and drug therapy trials aim to find treatments for retinal genetic disorders. ${ }^{7}$ These trials have been largely successful, in part because of several unique features of the eye. ${ }^{8}$ However, exactly which disorder causes patient phenotypes is often unclear because of a large degree of similarity between different retinal diseases and the broad range of phenotypes caused by mutations in a single gene. This makes genetic diagnosis a necessary first step toward personalized therapy.

Genotyping microarrays, such as the Asper Ophthalmics genomics chip, are typically used for genetic diagnosis of STGD and identify the cause of disease in $30-40 \%$ of patients. ${ }^{9}$ By contrast, a small number of studies using next-generation sequencing (NGS) display increased sensitivity of molecular diagnosis, largely because of their ability to detect novel disease alleles. ${ }^{10}$ To maintain the accuracy of NGS while reducing cost, capture protocols that enrich for DNA of interest, allowing targeted sequencing, have been developed.

Most cases of STGD are caused by recessive ABCA4 mutations. However, in many patients with STGD with an unknown cause only one disease-causing $A B C A 4$ mutation is detected. ${ }^{10}$ Deep intronic variants, deletions that escape genetic detection,

${ }^{1}$ Human Genome Sequencing Center, Baylor College of Medicine, Houston, Texas, USA; ${ }^{2}$ Department of Molecular and Human Genetics, Baylor College of Medicine, Houston, Texas, USA; ${ }^{3}$ McGill Ocular Genetics Laboratory, Montreal Children's Hospital, Departments of Paediatric Surgery, Human Genetics and Ophthalmology, McGill University Health Centre, Montreal, Quebec, Canada; ${ }^{4}$ Department of Ophthalmology, Peking Union Medical College Hospital, Peking Union Medical College, Beijing, China; ${ }^{5}$ Structural and Computational

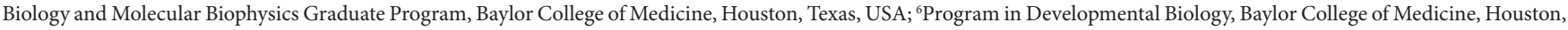
Texas, USA. Correspondence: Rui Chen (ruichen@bcm.edu) or Robert Koenekoop (robkoenekoop@hotmail.com) 
or a currently unknown STGD gene may explain these cases. Our aims in this study were to use NGS to assess the spectrum of $A B C A 4$ mutations in our patient cohort and to examine patients for disease-causing mutations in other retinal disease genes.

Here we present results from highly accurate capture panel sequencing of all known retinal disease genes, leading to an overall molecular diagnosis rate of $67 \%$ in our cohort, consisting of 18 French Canadian (FC), 39 other Canadian, and 31 Chinese patients. More than half (53\%) of the cases of disease were explained by homozygous or compound heterozygous mutations in $A B C A 4$. Among the 88 individuals examined in this study, we identified 35 novel $A B C A 4$ mutations. Whereas the results for the Chinese and Canadian cohorts were similar, our FC cohort had a statistically significantly reduced solving rate, indicating that unidentified mutations are enriched in this population. Finally, we identified causative mutations currently associated only with other macular dystrophies, not clinical STGD. This may be due to an incorrect initial clinical diagnosis or genetic heterogeneity of the disease. Either way, this emphasizes the importance of sequencing all known retinal disease genes when performing molecular diagnosis of STGD.

\section{MATERIALS AND METHODS}

Two cohorts of patients were examined. The first STGD cohort was from China, recruited by R.S., whereas the second contained individuals recruited in Canada by R.K.

\section{Clinical evaluation: Chinese patients}

Probands and other family members were ascertained primarily at Peking Union Medical College Hospital (Beijing, China). Medical and family histories were recorded. Detailed ophthalmologic examinations, including visual acuity, color vision test (pseudoisochromatic plates and D-15 color plates), slit lamp biomicroscopy, tonometry, and dilated ophthalmoscopy, were conducted. Macular structure was examined with optical coherence tomography (OCT) (3D OCT-2000 Spectral Domain; Topcon, Tokyo, Japan). Autofluorescence images (HRA 1; Heidelberg Engineering, Heidelberg, Germany) were obtained. Full-field electroretinograms (ERGs) were obtained (RetiPort ERG system; Roland Consult, Wiesbaden, Germany) in selected patients. The method was performed in concordance with the International Society for Clinical Electrophysiology of Vision standard protocol.

Diagnosis of STGD was based on clinical manifestations. Written informed consent was obtained from participants or their guardians. Genomic DNA was isolated from peripheral leukocytes using a commercial kit (QIAamp Blood Midi Kit; Qiagen, Hilden, Germany) according to the manufacturer's protocol. This study was approved by the institutional review board of Peking Union Medical College Hospital and adhered to the tenets of the Declaration of Helsinki and the Guidance on Sample Collection of Human Genetic Diseases by the Ministry of Public Health of China.

\section{Clinical evaluation: Canadian patients}

All patients were seen at Montreal Children's Hospital McGill Ocular Genetics Laboratory and informed consent was obtained. All patients underwent a detailed history, pedigree analysis, and detailed eye examinations, including best-corrected visual acuity using projected Snellen charts, near vision, slit lamp biomicroscopy, and dilated retinal exams. In vivo retinal imaging was performed using a Heidelberg OCT device (Heidelberg Engineering), followed by fundus autofluorescence (FAF); because imaging began in 2009, some patients did not receive this testing until after their molecular diagnosis was confirmed. Kinetic fields were measured by Goldmann perimetry. In selected patients we obtained International Society for Clinical Electrophysiology of Vision standard ERGs and multifocal ERGs (Diagnosys, Boston, MA). Clinical diagnosis of STGD was made when the patient, usually a child, developed central visual acuity loss with an atrophic maculopathy with or without flecks. Peripheral blood was collected in lavender-top (EDTA) tubes for DNA extraction.

\section{Clinical evaluation: comparison}

The clinician authors of this study (R.S. and R.K.) actively collaborated to control for differences in diagnosis between the cohorts. This involved both clinicians reviewing and coming to a consensus on clinical data in some cases for which the diagnosis was in question. The two clinicians used largely identical visual acuity tests, slit lamp biomicroscopy, dilated ophthalmoscopy, and OCT in all patients when generating a clinical diagnosis. However, despite our effort to maintain consistent criteria, there were slight differences between the two cohorts. Specifically, ERGs were performed on all patients in the Chinese cohort but only in selected patients in the Canadian cohort, whereas FAF was performed on all Canadian patients but only some Chinese patients.

\section{Capture sequencing and data analysis}

Patient DNA was extracted from peripheral blood using standard techniques. A precapture library was generated ${ }^{11}$ and then captured on a custom capture panel designed using Agilent Sureselect (Agilent Technologies, Santa Clara, CA) and targeting all known retinal disease genes (Supplementary Table S1 online). Resultant DNA was bar-coded and prepared, then shotgun sequenced on an Illumina GIIx machine (http://support. illumina.com/sequencing/sequencing_instruments/genome_ analyzer_iix.html). Reads were aligned to hg19 using Burrows Wheeler Aligner. ${ }^{12}$ Recalibration and realignment were performed using the Genome Analysis Toolkit. ${ }^{13}$ SAMtools ${ }^{14}$ was used to sort and index the resulting bam files. Single-nucleotide polymorphisms and insertions/deletions were called using Atlas SNP and Atlas Indel, respectively. ${ }^{15}$

These variants were filtered and annotated using a suite of software. First, variants with $>0.5 \%$ frequency in the 1000 Genomes Project builds 2010 and 2011 (ref. 16), National Heart, Lung, and Blood Institute GO Exome Sequencing Project cohort, ${ }^{17}$ or an internal control database at the human genome sequencing center were filtered out. Second, variants 
that occurred at a frequency of $>10 \%$ in our patient cohort were filtered out because they are likely systematic errors. Third, variants were annotated using ANNOVAR. ${ }^{18}$ Variants not affecting protein sequence or splicing were removed. Finally, variants were annotated with predictions from SIFT, ${ }^{19}$ POLYPHEN $2,{ }^{20}$ and various other annotation programs using dbNSFP version 2.0b4. ${ }^{21}$ Final results were manually verified on up-to-date online tools for HGMD, ${ }^{22}$ SIFT, and POLYPHEN2, accessed 17 February 2014.

\section{Sanger verification and intronic sequencing}

All reported causative mutations were confirmed by Sanger sequencing using standard techniques. Segregation was performed when possible. All patients that had an unsolved disease cause after capture sequencing were tested for causative intronic mutations. Specifically, we tested for the seven intronic mutations described by Braun et al., ${ }^{23}$ as well as sequencing retinal-specific exons reported by Farkas et al. ${ }^{24}$ Primers and Sanger sequencing tracks are available upon request.

\section{Molecular diagnosis}

If at least two variants in $A B C A 4$ that passed filtering criteria were found, the variants were considered as candidate diseasecausing mutations. In all cases in which a novel $A B C A 4$ mutation was found, no other set of mutations in the patients that is known to be sufficient to cause retinal disease was found. Patients whose disease was unexplained by $A B C A 4$ variants were examined for mutations known to cause other retinal diseases.

\section{Copy-number variation analysis}

Copy-number variation (CNV) analysis was performed using a custom-designed comparative genomic hybridization microarray (Agilent Technologies). The high-density, $8 \times 60-\mathrm{K}$ array covers known STGD genes and genes associated with agerelated macular dystrophy. The designed probe density was $>50$ oligos/kb. The digestion, labeling, and hybridization processes were performed according to the manufacturer's instructions. Array slides were scanned with the Agilent G2565 microarray scanner. Resulting images were quantified using Agilent Feature Extraction software and analyzed using Agilent Genomic Workbench (http://www.genomics.agilent.com).

\section{RESULTS}

We designed and tested new capture sequencing reagents targeting all known exons of all known retinal disease genes (at time of design, 13 December 2012). Our panel was designed using Agilent SureSelect and captures 213 known retinal disease genes. All genes included and a justification for their relevance to retinal disease can be found in the Supplementary Data online.

This capture panel was applied to 88 patients with an original diagnosis of STGD, including 57 patients examined at the Montreal Children's Hospital in Montreal, Canada, and 31 patients examined at Peking Union Medical College Hospital in China. Rigorous quality control was performed, examining the total number of reads, percentage of reads mapping to the target, coverage in the target region, and number of variants observed before and after filtering. Experiments with poor data quality were repeated. On average, $98.86 \%$ of the target was covered; $92.86 \%$ of the target had coverage $>10 \times$, which is sufficient to call heterozygous mutation $s^{25}$ (Figure 1).

After verifying the quality of our sequencing results, we examined our patients for pathogenic mutations in $A B C A 4$. All mutations were Sanger verified. In addition, patients whose disease could not be explained by capture sequencing alone were checked for known disease-causing intronic mutations in $A B C A 4$. Three patients (patients 27, 28, and 52) had mutation V1 described by Braun et al. ${ }^{23}$ Patient 28 also had an observed novel exonic hit and is thus considered solved. No mutations at the positions of V2-V7 identified by Braun et al were observed. No predicted pathogenic mutations in the retinal-specific exons identified by Farkas et al ${ }^{24}$ were found.

STGD in 48 patients (55\%) was explained by recessive $A B C A 4$ mutations (Supplementary Table S2 online). Only 22 patients could be solved using previously known STGD-causing mutations. However, we identified 35 novel mutations in $A B C A 4$ contributing to the diagnosis of 25 patients with STGD. This contained 10 novel mutations leading to amino acid substitutions already known to cause disease and mutations known to cause diseases other than STGD. In addition, we identified 13 novel nonsense mutations. The remaining 12 novel mutations are well justified, and novel mutations were either completely absent or extremely rare in controls.

Interestingly, whereas we found disease-causing $A B C A 4$ mutations in $61 \%(19 / 31)$ and $59 \%(23 / 39)$ of our Chinese and Canadian cohorts, respectively, only $33 \%(6 / 18)$ of our FC cohort was resolved by leveraging $A B C A 4$ mutations. This result is statistically significant $(P<0.05$ versus Chinese and Canadian cohorts using Fisher exact test). This indicates that one or more unidentified founder STGD-causing mutations may exist in this FC population, resulting in the low rate of molecular diagnosis. Founder mutation(s) could occur in $A B C A 4$ exons

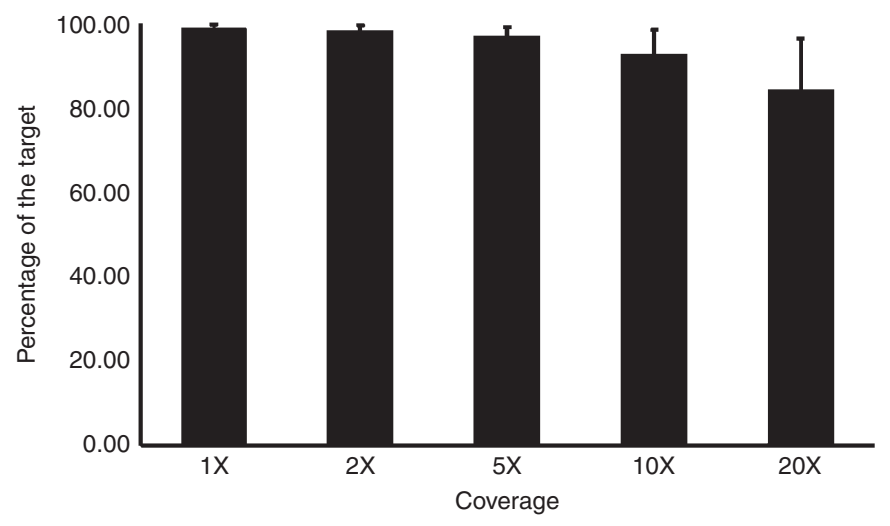

Figure 1 High-quality sequencing results were obtained, with consistently high coverage across the targeted region. Almost the entire target was covered sufficiently to call single-nucleotide polymorphisms. Error bars display $1 \mathrm{SD}$. 
Table 1 Disease in 11 patients explained by mutations outside $A B C A 4$

\begin{tabular}{|c|c|c|c|c|c|c|}
\hline ID/cohort & Gene & Ref-Seq ID & Genotype & cDNA & Protein & Justification \\
\hline 13/Can & PRPH2 & NM_000322 & Heterozygous & c. $37 \mathrm{C}>\mathrm{T}$ & p.(R13W) & Known (RP) $)^{34}$ \\
\hline \multirow[t]{2}{*}{ 55/FC } & CRB1 & NM_001193640 & Compound & c. $635 \mathrm{G}>\mathrm{A}$ & p.(C212Y) & SIFT, PolyP \\
\hline & & & Heterozygous & c. $2507 \mathrm{G}>\mathrm{A}$ & p.(C836Y) & Known AA (RP) ${ }^{35}$ \\
\hline $57 / F C$ & PRPH2 & NM_000322 & Heterozygous & c. 499 G>A & p.(G167S) & Known $(P D)^{26,36}$ \\
\hline 61/Can & $D M D$ & NM_004010 & Homozygous & c. 2458 C>T & p.(R820C) & SIFT, PolyP, H \\
\hline 62/Can & USH2A & NM_206933 & Compound & c. $5953 \mathrm{G}>\mathrm{A}$ & p.(E1985K) & PolyP \\
\hline 70/Can & $\mathrm{CDH} 23$ & NM_001171930 & Homozygous & c. 2263 C>T & p.(H755Y) & Known (USH1) $)^{38-40}$ \\
\hline \multirow[t]{2}{*}{$80 / F C$} & CRB1 & NM_001193640 & Compound & c. $3350 \mathrm{G}>\mathrm{C}$ & p.(C1117S) & SIFT, PolyP \\
\hline & & & Heterozygous & c.493_501del & 165_167del & 0 \\
\hline 96/Can & $\mathrm{CFH}$ & NM_000186 & Homozygous & c. $101 \mathrm{C}>\mathrm{G}$ & p.(T34R) & SIFT \\
\hline
\end{tabular}

Patients who were clinically examined following the molecular diagnosis are in bold. Under Justification, "Known AA" indicates that the amino acid substitution is known to cause disease, whereas "Known" indicates this specific DNA change is known to cause disease. The disease the mutation was associated with follows in parentheses. All novel mutations had a frequency $<0.002$ in a control cohort of 6,500 individuals (ESP6500). For novel mutations, use the following key: SIFT, predicted damaging by SIFT; PolyP, predicted damaging by Polyphen 2; Splice, predicted splice-site loss mutation; STGD, this gene is known to cause Stargardt-like phenotypes; O, overlaps with known disease causing missense and nonframeshift deletion mutations.

Can, other Canadian; CDNA, complementary DNA; Chi, Chinese; FC, French Canadian; PD, pattern dystrophy; RP, retinitis pigmentosa; USH1, Usher syndrome type 1.

or introns or in other retinal disease genes. Alternatively, the FC population may have a different mutation spectrum than other cohorts. The FC population did not show a statistically significant increase in unsolved patients with a single observed mutation in $A B C A 4$. This was because of a lack of power; only eight FC patients are unsolved. The remaining four FC patients have disease likely caused by mutations in other retinal disease genes (Table 1).

Surprisingly, 13/29 (45\%) unsolved patients had a single likely pathogenic mutation in $A B C A 4$ and no second $A B C A 4$ mutation found, which is significantly higher than the expected rate among the general population (5\%). This effect had no significant variation between cohorts. To investigate whether CNVs in $A B C A 4$ could explain some of these unsolved cases, we performed chromosomal analysis on the 12 patients with a single heterozygous hit in ABCA4. No likely deleterious CNVs were observed (Figure 2). Those patients who were not solved following thorough analysis of $A B C A 4$ were checked for mutations in other retinal disease genes based on capture sequence data. Eleven patients (13\%) were solved by invoking retinal disease genes not previously linked to STGD (Table 1), bringing the total number of molecularly diagnosed patients to 59 (67\%). Eleven novel candidate disease-causing mutations outside of $A B C A 4$ were found (Table 1).

We wished to determine whether patients with mutations outside $A B C A 4$ had an initial clinical misdiagnosis or whether mutations in these nine genes can cause STGD-like phenotypes. To this end, two patients harboring mutations outside $A B C A 4$ were reexamined in detail. Clinically, these subjects (Figures 3 and 4) had characteristic STGD visual acuity loss and an atrophic maculopathy with retinal flecks and central scotomas on

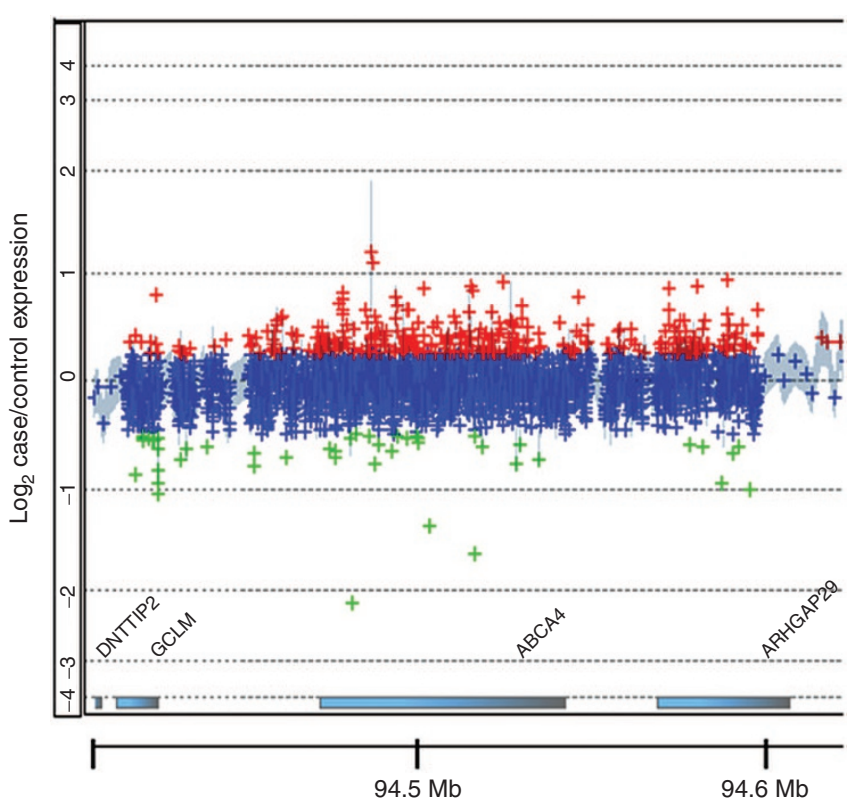

Figure 2 Representative comparative genomic hybridization microarray from a patient with one hit in $A B C A 4$. Despite a very high probe density, no significant copy-number variations were found in any of the 12 patients with one mutation in $A B C A 4$.

Goldmann visual fields (GVFs). Both patients had been seen by several ophthalmologists, retinal specialists, or pediatric ophthalmologists, and the consensus clinical diagnosis was STGD. Upon molecular testing we first ruled out $A B C A 4$ mutations, then identified two likely pathogenic mutations in the Bestrophin-1 (BEST1) and Crumbs homolog 1 (CRB1) genes, respectively. 

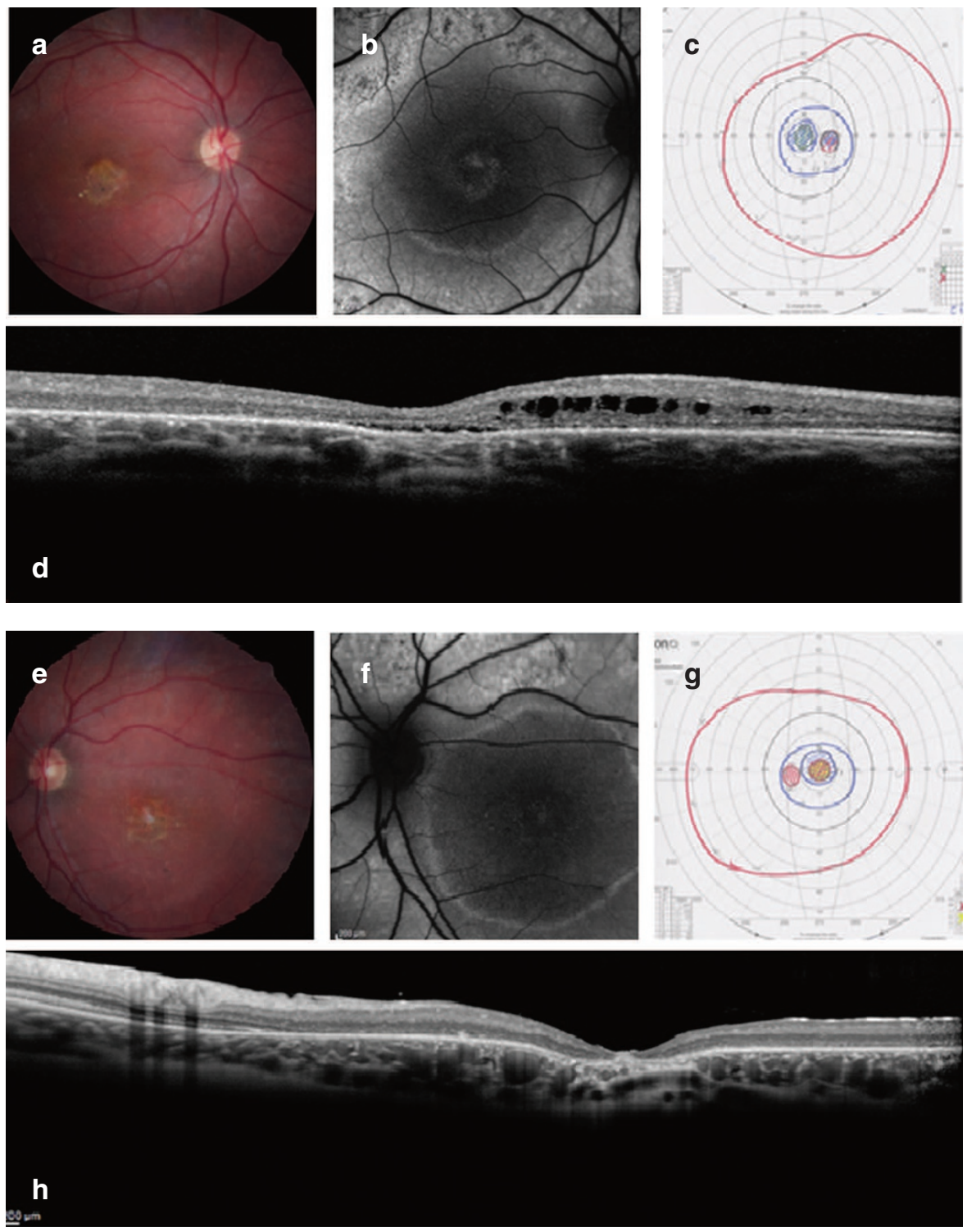

Figure 3 Patient with a homozygous BEST1 mutation presents a STGD phenotype. (a,e) Fundus photographs of patient 69 show a bilateral atrophic maculopathy with yellow retinal flecks, as well as retinal vasculature and optic discs that are within normal limits. (b,f) Fundus autofluorescence images show an incomplete rim of hyperfluorescence in the right macular area and a complete hyperfluorescent ring in the left macula. (c,g) Goldmann visual field shows central scotoma in both eyes. (d, h) Optical coherence tomography shows loss of the inner segment/outer segment junctions, a disorganized external limiting membrane and outer nuclear layer, thinning of the outer nuclear layer in the fovea, and subfoveal edema. There were marked cystic spaces in the inner nuclear layer.

\section{Clinical phenotypes of three unusual patients with unexpected clinical rediagnoses}

Subject 69 , with a homozygous BEST1 mutation, is a 33-yearold woman from the Mohawk nation of Montreal, Quebec. She presented with a progressive decrease in central vision. Her bestcorrected visual acuity was 20/70 in the right eye and 20/400 in the left eye. Her problem started at 13 years of age. Her parents were nonconsanguineous but from a small and ancient original Canadian community. The proband's brother also lost central vision in his 20s. The proband had a peripheral iridectomy for glaucoma in both eyes. Clinically, the patient's ocular phenotype was STGD (diagnosed by three retinal specialists) but her molecular diagnosis was autosomal recessive bestrophinopathy because she carries homozygous BEST1 mutations. Figure 3 shows fundus photographs, OCT and FAF images, and GVFs that were obtained after the molecular diagnosis. The patient's phenotype consists of an atrophic macular lesion, subfoveal and parafoveal retinal edema, loss of the inner segment/outer segment junction in the macula, a perifoveal hyperfluorescent ring on FAF, and central scotomas on GVFs.

Subject 55 is a 23 -year-old FC woman from Quebec who presented with progressive central vision loss at age 5 and has compound heterozygous mutations in CRB1. Her best-corrected visual acuity was 20/400 in the right eye and 20/50 in the left eye. She denied nyctalopia. Her parents were also from Quebec and nonconsanguineous, although that the Quebec population 
has strong founder effects is well known. Her clinical phenotype was STGD with characteristic atrophic maculopathy and retinal flecks. Retinal vasculature and peripheral retina were within normal limits in both eyes. Molecular testing results showed that the proband carries compound heterozygous $C R B 1$ mutations: p.Cys212Tyr/p.Cys948Tyr and c.635G>A/c.2843G>A.
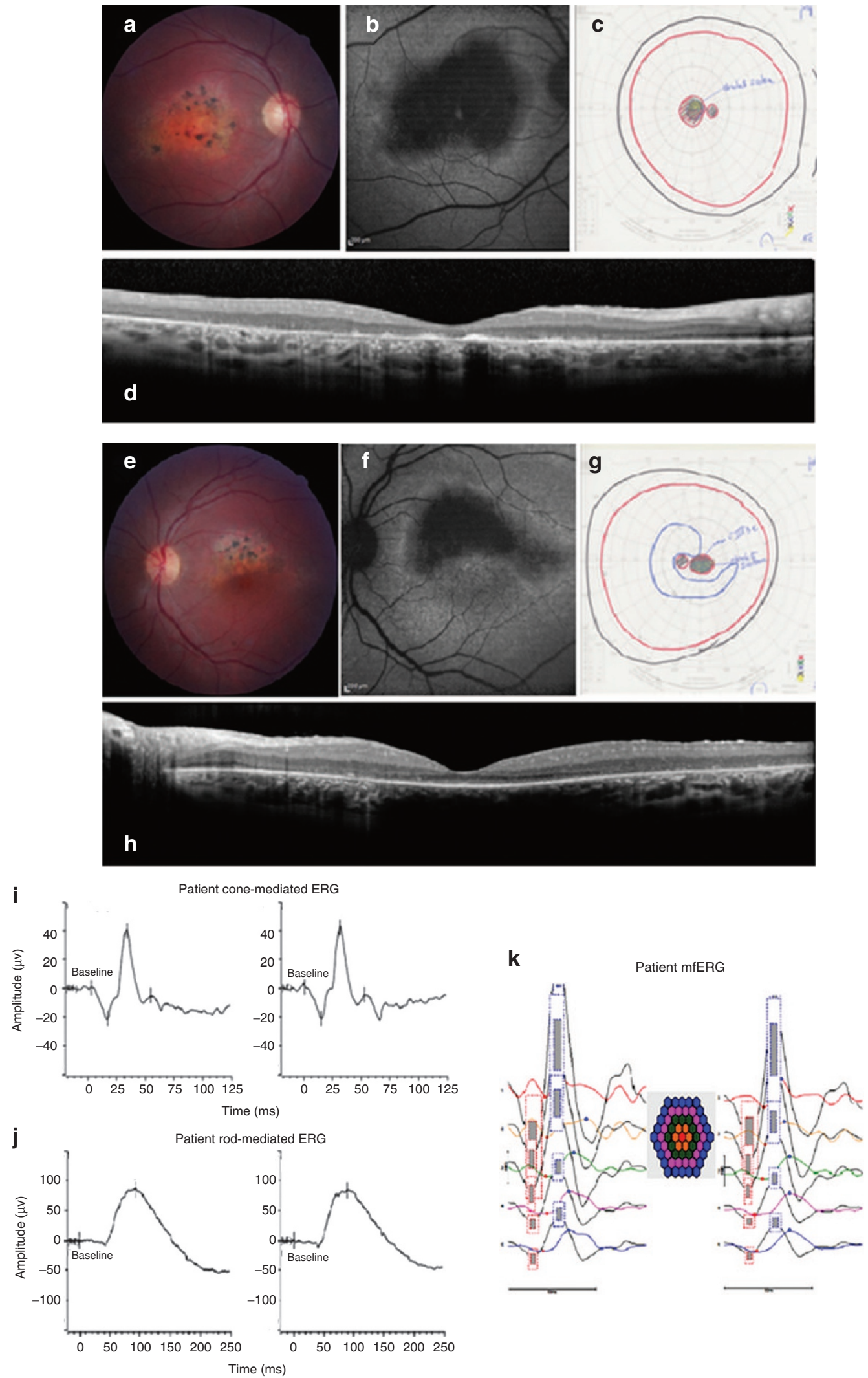
The C212Y mutation in CRB1 is a novel splicing mutation, leading to a loss of that exon and a frameshift in the CRB1 protein, whereas the C948T mutation was previously reported to cause RP.

After genotyping, ERG was performed at age 23, which was essentially normal for both rod and cone function. It showed that the rod-mediated ERG b-wave was within normal amplitude and peak time limits in both eyes (Figure 4). The rod-conemediated ERG a- and b-wave amplitudes were within normal limits (both eyes), a-wave peak times were within normal limits (both eyes), and b-wave peak times were significantly delayed (both eyes). The cone-mediated ERG a-waves were within normal amplitudes in both eyes and b-waves were within lower limits of normal amplitude. This is an unusual ERG for a patient with CRB1 mutations. Figure 4 shows patient fundus photographs, OCT and FAF images, GVFs, and focal and multifocal ERGs. This illustrates an atrophic maculopathy, a large central hypofluorescence on FAF, loss of inner segment/outer segment junctions in the macula on OCT, and central scotomas on GVF. The ERG is significant for a patient with CRB1 mutations in that it is essentially intact.

The other patient with CRB1 mutations (patient 80; Table 1) presented with visual acuity loss in the left eye at 8 years old and visual acuity loss in the right eye at age 45 . We observed bull'seye maculopathy with retinal flecks in both eyes, 20/50 visual acuity in the right eye and counting fingers in the left eye, and an essentially normal full-field cone and rod ERG. The patient tested normal for GVFs. Based on these data, the patient was diagnosed with STGD. FAF showed central hypofluorescence, and OCT showed severe foveal thinning. In conclusion, the two patients with CRB1 mutations have similar phenotypes, both of which fall within the standard clinical definition of STGD. In addition, it is interesting to note that this is not the first report of CRB1 mutations causing disease in a patient originally diagnosed with STGD. In accord with our patients, this patient had more severe degeneration in one eye, along with severe central vision impairment occurring at age 37 , although, unlike our patients, no fundus flecks were observed. ${ }^{26}$ Taken together, these data suggest a novel phenotype associated with CRB1 mutations.

\section{DISCUSSION}

This study demonstrates the ability of capture sequencing to increase the rate of molecular diagnosis in a cohort of patients with clinically diagnosed STGD, a vital first step toward therapy and personalized medicine. We identified the molecular cause of disease in 59 of our 88 patients, achieving a rate of $67 \%$. The increase in accuracy we obtained over traditional methods is due to three features of our capture sequencing protocol, which allowed for a comprehensive analysis of the $A B C A 4$ locus and, when this failed to identify the cause of disease, other retinal disease genes. First, because we sequenced the entire coding region of $A B C A 4$, we were able to identify novel STGD-causing mutations. This feature contributed to the diagnosis of 25 (28\%) of our patients. Second, our capture technique allowed us to identify mutations in other known retinal disease genes, allowing for correction in cases of unclear diagnosis or misdiagnosis and allowing us to identify novel phenotype-genotype correlations. This feature contributed to the diagnosis of $11(13 \%)$ of our patients. Third, we tested for recently identified intronic mutations in $A B C A 4$ and found three such mutations in our patient cohort. The candidate novel mutations identified by this study are well supported.

Numerous other recent papers have noted that there is much overlap between genes causing different forms of retinal disease. ${ }^{27-29}$ These recent findings support the conclusion that there is no simple connection between the gene in which a mutation occurs and a patient's retinal phenotype, making strict categorization of genetic disease complicated. Furthermore, this observation indicates a thorough description of any patient's disease should include both genetic and clinical information. The high percentage of patients harboring novel alleles and mutations in genes not previously associated with STGD also demonstrates the strength of NGS as a molecular diagnostic tool. All novel mutations and all mutations outside of $A B C A 4$ would have been missed by a standard diagnostic genotyping array, reducing our molecular diagnosis rate by $40 \%$. In addition, our approach identified interesting phenotype-genotype correlations.

In particular, two patients were diagnosed by multiple retinal and pediatric ophthalmology specialists with STGD but had disease-causing mutations outside $A B C A 4$. These patients were reexamined clinically. The genetic testing allowed us to refine their diagnosis and reclassify them into new clinical diagnostic categories. Patient 69 was diagnosed with STGD but instead had autosomal bestrophinopathy due to recessive BEST1 mutations. Ocular phenotypes associated with mutations in BEST1 include $\mathrm{RP}$, rod-cone dystrophy, and bestrophinopathy, ${ }^{30}$ changing the differential diagnosis of this patient.

In the second case we found CRB1 mutations in a child with maculopathy, central scotomas, and an essentially robust and normal ERG (patient 55). CRB1 mutations cause severe diffuse retinal degeneration with absent or severely diminished ERGs, including Leber congenital amaurosis, juvenile RP, and conerod dystrophy. To our knowledge this is the first case of a retinal

Figure 4 Patient with compound heterozygous CRB1 mutations presents a STGD phenotype. (a,e) Fundus photographs of patient 55 show an atrophic maculopathy involving the fovea in the right eye with clumps of pigmentation in the macular area. The left eye shows an atrophic maculopathy sparing the inferior half of the fovea. Retinal vessels and optic discs are within normal limits. (b,f) Fundus autofluorescence images show macular atrophy that appears dark black and involves the fovea in the right eye (OD); in the left eye (OS) the inferior half of the fovea is spared. (c, $\mathbf{g}$ ) Goldmann visual fields show an absolute central scotoma in both eyes (OU). (d,h) Optical coherence tomography shows bilateral loss of inner segment/outer segment junctions and decreased retinal thickness. Accumulation of autofluorescent photoreceptor debris in the foveal area is seen in the OD. (i) Cone-mediated electroretinography (ERG) a-waves are within normal amplitude limits (OU) and peak times are delayed (OD) and within normal limits (OS). The b-waves are within the lower limits of normal amplitude (OU), with peak times that are delayed (OD) and within normal limits (OS). (j) The rod-mediated ERG b-wave is within normal amplitude and peak time limits (OU). (k) Multifocal ERGs are attenuated in amplitude at all eccentricities. 
dystrophy caused by CRB1 mutation with a normal ERG; CRB1 mutations usually lead to severe RP or Leber congenital amaurosis with extinguished or severely diminished ERGs. There are certain specific retinal findings (including preserved paraarteriole retinal pigment epithelium and nummular pigment) that indicate CRB1 mutations are causative, yet these were not found in this patient. Largely similar results were observed in a second patient with CRB1 mutations. These data demonstrate a novel CRB1 phenotype. Given that overlapping phenotypes are caused by mutations in different genes and many genes cause a spectrum of phenotypic outcomes, the current best way to determine the genetic cause of disease is through capture sequencing.

The enrichment for patients with a single hit in the recessive disease gene $A B C A 4$ indicates that $A B C A 4$ is likely involved in the disease of many of our undiagnosed patients. Exonic regions are well covered by our method, and despite examining a number of patients with high-density array comparative genomic hybridization, no CNVs were observed. This leads to the exciting possibility that novel types of mechanisms (regulatory mutations, cryptic splice-site formation, or digenic effects) are contributing to the genetic cause of disease in our patients. Indeed, a recent study identified numerous altered transcripts in $A B C A 4$ as a result of splicing modifications and synonymous variants. ${ }^{23}$ Alternatively, these $A B C A 4$ mutations may act in a digenic or multigenic fashion to cause disease.

Comparison of our three cohorts showed surprising similarity between the Chinese and Canadian populations. However, a paucity of disease-causing $A B C A 4$ mutations was observed in our FC cohort. This effect is statistically significant and raises the possibility of currently unknown founder mutation(s) in this cohort, which may occur in the exons or introns of $A B C A 4$ or another gene. Patients of FC origin from Quebec are known to come from a gene pool with the potential for founder effects; most of the 8 million people living in the province today are descendants of 2,500 French forefathers, mostly from northern France. Further studies targeting FC patients with STGD are thus recommended to elucidate the reason for this missing inheritance.

Several recent studies also performed NGS-based genetic diagnosis of patients with STGD. ${ }^{10,31-33}$ The central conclusions of these studies are that $A B C A 4$ holds additional pathogenic mutations that are missed during exon sequencing and that NGS significantly improves the accuracy of genetic diagnosis. Given the increase in accuracy of capture sequencing over array-based diagnosis, health-care institutions should adopt this new technology whenever possible. Unfortunately, several obstacles remain, including methodologies to handle variants of unknown significance and standardized pipelines and metrics to ensure repeatability and reliability of data. Nonetheless, the increase in accuracy afforded by capture sequencing will make it an invaluable tool in the development of personalized medicine. This study represents a first step toward treatment for two-thirds of our patients by identifying the genetic cause of their disease, giving them the potential participate in developing genetic therapy techniques.

\section{SUPPLEMENTARY DATA}

Supplementary material is linked to the online version of the paper at $h$ ttp://www.nature.com/gim

\section{ACKNOWLEDGMENTS}

The authors thank all patients, parents, and families involved in this study. They also thank James R. Lupski and Phillip Michael Boone for their assistance with the microarray analysis. J.Z. is supported by a training fellowship from the Keck Center of the Gulf Coast Consortia, on the NLM Training Program in Biomedical Informatics (NLM grant T15LM007093). R.C. is supported by grants from Retinal Research Foundation, Foundation Fighting Blindness (BR-GE0613-0618-BCM), and the National Eye Institute (R01EY022356, R01EY018571). R.K. is supported by Foundation Fighting Blindness, Canada; Canadian Institutes of Health Research; Fonds de la Recherche en Santé du Québec; and National Institutes of Health. R.S. is supported by the Ministry of Human Resource and Social Security of the People's Republic of China and the Foundation Fighting Blindness, USA (CD-CL-0808-0470-PUMCH). F.W. is supported by a predoctoral fellowship funded by the Burroughs Wellcome Trust Fund: The Houston Laboratory and Population Sciences Training Program in Gene Environment Interaction.

\section{DISCLOSURE}

The authors declare no conflict of interest.

\section{REFERENCES}

1. Burke TR, Tsang SH. Allelic and phenotypic heterogeneity in ABCA4 mutations. Ophthalmic Genet 2011;32:165-174.

2. Molday RS, Zhang K. Defective lipid transport and biosynthesis in recessive and dominant Stargardt macular degeneration. Prog Lipid Res 2010;49:476-492.

3. Kaplan J, Gerber S, Larget-Piet D, et al. A gene for Stargardt's disease (fundus flavimaculatus) maps to the short arm of chromosome 1. Nat Genet 1993;5:308-311.

4. Yang Z, Chen Y, Lillo C, et al. Mutant prominin 1 found in patients with macular degeneration disrupts photoreceptor disk morphogenesis in mice. J Clin Invest 2008;118:2908-2916

5. Zhang K, Kniazeva M, Han M, et al. A 5-bp deletion in ELOVL4 is associated with two related forms of autosomal dominant macular dystrophy. Nat Genet 2001;27:89-93.

6. Kjellström U. Association between genotype and phenotype in families with mutations in the ABCA4 gene. Mol Vis 2014;20:89-104.

7. Bennett J, Ashtari M, Wellman J, et al. AAV2 gene therapy readministration in three adults with congenital blindness. Sci Trans/ Med 2012;4:120ra15.

8. Zaneveld J, Wang F, Wang X, Chen R. Dawn of ocular gene therapy: implications for molecular diagnosis in retinal disease. Sci China Life Sci 2013;56:125-133.

9. Ernest PJ, Boon CJ, Klevering BJ, Hoefsloot LH, Hoyng CB. Outcome of ABCA4 microarray screening in routine clinical practice. Mol Vis 2009;15:2841-2847.

10. Fujinami K, Zernant J, Chana RK, et al. ABCA4 gene screening by nextgeneration sequencing in a British cohort. Invest Ophthalmol Vis Sci 2013;54: 6662-6674.

11. Koenekoop RK, Wang H, Majewski J, et al.; Finding of Rare Disease Genes (FORGE) Canada Consortium. Mutations in NMNAT1 cause Leber congenital amaurosis and identify a new disease pathway for retinal degeneration. Nat Genet 2012;44:1035-1039.

12. Li H, Durbin R. Fast and accurate short read alignment with Burrows-Wheeler transform. Bioinformatics 2009;25:1754-1760

13. McKenna A, Hanna M, Banks E, et al. The Genome Analysis Toolkit: a MapReduce framework for analyzing next-generation DNA sequencing data. Genome Res 2010;20:1297-1303.

14. Li H, Handsaker B, Wysoker A, et al.; 1000 Genome Project Data Processing Subgroup. The Sequence Alignment/Map format and SAMtools. Bioinformatics 2009;25:2078-2079. 
15. Shen $Y$, Wan $Z$, Coarfa $C$, et al. A SNP discovery method to assess variant allele probability from next-generation resequencing data. Genome Res 2010;20:273-280.

16. Abecasis GR, Auton A, Brooks LD, et al. An integrated map of genetic variation from 1,092 human genomes. Nature 2012;491:56-65.

17. Peloso GM, Auer PL, Bis JC, et al.; NHLBI GO Exome Sequencing Project. Association of low-frequency and rare coding-sequence variants with blood lipids and coronary heart disease in 56,000 whites and blacks. Am J Hum Genet 2014;94:223-232.

18. Wang K, Li M, Hakonarson H. ANNOVAR: functional annotation of genetic variants from high-throughput sequencing data. Nucleic Acids Res 2010;38:e164.

19. Kumar P, Henikoff S, Ng PC. Predicting the effects of coding non-synonymous variants on protein function using the SIFT algorithm. Nat Protoc 2009;4:10731081.

20. Adzhubei IA, Schmidt S, Peshkin L, et al. A method and server for predicting damaging missense mutations. Nat Methods 2010;7:248-249.

21. Liu X, Jian X, Boerwinkle E. dbNSFP: a lightweight database of human nonsynonymous SNPS and their functional predictions. Hum Mutat 2011;32:894-899.

22. Stenson PD, Ball EV, Mort M, et al. Human Gene Mutation Database (HGMD): 2003 update. Hum Mutat 2003;21:577-581.

23. Braun TA, Mullins RF, Wagner AH, et al. Non-exomic and synonymous variants in $A B C A 4$ are an important cause of Stargardt disease. Hum Mol Genet 2013;22:5136-5145.

24. Farkas MH, Grant GR, White JA, Sousa ME, Consugar MB, Pierce EA. Transcriptome analyses of the human retina identify unprecedented transcript diversity and $3.5 \mathrm{Mb}$ of novel transcribed sequence via significant alternative splicing and novel genes. BMC Genomics 2013;14:486.

25. Sulonen AM, Ellonen $\mathrm{P}, \mathrm{Almusa} \mathrm{H}$, et al. Comparison of solution-based exome capture methods for next generation sequencing. Genome Bio/ 2011;12:R94.

26. Strom SP, Gao YQ, Martinez A, et al. Molecular diagnosis of putative Stargardt disease probands by exome sequencing. BMC Med Genet 2012;13:67.

27. Wang X, Wang H, Sun V, et al. Comprehensive molecular diagnosis of 179 Leber congenital amaurosis and juvenile retinitis pigmentosa patients by targeted next generation sequencing. J Med Genet 2013;50:674-688.
28. Wang $F$, Wang $H$, Tuan $H F$, et al. Next generation sequencing-based molecular diagnosis of retinitis pigmentosa: identification of a novel genotype-phenotype correlation and clinical refinements. Hum Genet 2014;133:331-345.

29. Fu Q, Wang F, Wang $H$, et al. Next-generation sequencing-based molecular diagnosis of a Chinese patient cohort with autosomal recessive retinitis pigmentosa. Invest Ophthalmol Vis Sci 2013;54:4158-4166.

30. Boon CJ, van den Born LI, Visser L, et al. Autosomal recessive bestrophinopathy: differential diagnosis and treatment options. Ophthalmology 2013;120:809820.

31. Zhang X, Ge X, Shi W, Huang P, Min Q, et al. Molecular diagnosis of putative Stargardt disease by capture next generation sequencing. PLOS One 2014;9(4):e95528. doi:10.1371/journal.pone.0095528.

32. Zernant J, Xie YA, Ayuso C, et al. Analysis of the ABCA4 genomic locus in Stargardt disease. Hum Mol Genet 2014; e-pub ahead of print 31 July 2014.

33. Jin X, Qu LH, Meng XH, Xu HW, Yin ZQ. Detecting genetic variations in hereditary retinal dystrophies with next-generation sequencing technology. $\mathrm{Mol}$ Vis 2014;20:553-560

34. Jacobson SG, Cideciyan AV, Kemp CM, Sheffield VC, Stone EM. Photoreceptor function in heterozygotes with insertion or deletion mutations in the RDS gene. Invest Ophthalmol Vis Sci 1996;37:1662-1674.

35. Henderson RH, Mackay DS, Li Z, et al. Phenotypic variability in patients with retinal dystrophies due to mutations in CRB1. Br J Ophthalmol 2011;95:811817.

36. Glöckle N, Kohl S, Mohr J, et al. Panel-based next generation sequencing as a reliable and efficient technique to detect mutations in unselected patients with retinal dystrophies. Eur J Hum Genet 2014;22:99-104.

37. Xu W, Dai H, Lu T, Zhang X, Dong B, Li Y. Seven novel mutations in the long isoform of the USH2A gene in Chinese families with nonsyndromic retinitis pigmentosa and Usher syndrome Type II. Mo/ Vis 2011;17:1537-1552.

38. Oshima A, Jaijo T, Aller E, et al. Mutation profile of the $\mathrm{CDH} 23$ gene in 56 probands with Usher syndrome type I. Hum Mutat 2008;29:E37-E46.

39. Bell CJ, Dinwiddie DL, Miller NA, et al. Carrier testing for severe childhood recessive diseases by next-generation sequencing. Sci Trans/ Med 2011;3:65ra4

40. Berg JS, Adams M, Nassar N, et al. An informatics approach to analyzing the incidentalome. Genet Med 2013;15:36-44. 